\title{
Molar Odontometrics In Gender Assessment - A Panoramic Radiographic Study.
}

\author{
Dr.Sitara Rathan ${ }^{1}$, Dr.Veena K M${ }^{2}$, Dr.Laxmikanth Chatra ${ }^{3}$, Dr.Prashanth \\ Shenoy ${ }^{4}$, Dr Rachana V Prabhu ${ }^{5}$
}

\begin{abstract}
:
Introduction: Identification of individuals, be it as part of criminal investigations or for any other purpose pertaining to public welfare like disaster management, is an integral part of forensic medicine. Dental tissues seldom get destroyed even in disastrous situations like arson. Moreover, various aspects of dental morphology have been shown to exhibit varying degrees of sexual dimorphism. This factor has often been employed in various studies in assessing the gender of an individual.

Aims And Objectives: This study primarily aimed to determine gender of an individual based on the MesioDistal (MD) dimensions of the permanent first mandibular molars on a panoramic radiograph and assess sexual dimorphism exhibited by the teeth.

Material And Methods: MD widths of the mandibular permanent first molars of 100 individuals from Yenepoya Dental College in Managalore were measured on panoramic radiographs by a digital scale and the data was subjected to relevant statistical analysis.

Results: The results revealed no evident significance in the mesiodistal width of the permanent mandibular $1^{\text {st }}$ molars between males and females.

Conclusion: Sexual dimorphism of teeth is population specific and the use of molar odontometrics via panoramic radiography hardly demonstrates any statistically significant sexual dimorphism.
\end{abstract}

Keywords:- sexual dimorphism, mandibular first molars, odontometrics, panoramic radiography, gender determination.

\section{Introduction}

Human identification is the recognition of an individual based on physical characteristics unique to that individual. Identification of human remains during mass disasters is often hindered by the state of the soft tissue. Hence several methods have been devised for identification from skeletal remains. ${ }^{1}$

The four main attributes of biological identification that the forensic anthropologists aim to determine are the gender, age, stature, and ethnic/racial background of the individual, of which gender determination is often the first step. This is because correct gender identification limits the pool of missing persons to just one-half of the population and also subsequent methods for age and stature estimation are often gender dependent. Among the various skeletal parts, skull and the pelvis are traditional indicators of gender and the accuracy rate of determining the gender correctly is nearly $100 \%$. $^{1}$

Teeth being the central element of the masticatory apparatus of skull, are fine sources of material for civil and medicolegal identification. Dental tissues seldom get destroyed even in disastrous situations like arson. Various aspects of dental morphology have been shown to exhibit varying degrees of sexual dimorphism. In addition, the degree to which they provide resistance to damage in terms of bacterial disintegration, fire and fracture, makes them valuable for forensic investigation and research. ${ }^{2,3,4,5}$ This factor has often been employed in various studies in assessing the gender of an individual. ${ }^{3}$ Though not as accurate as the skeleton, tooth crown dimensions are reasonably accurate predictors of gender and are useful adjuncts in gender assessment. Also this is of special importance in young individuals where skeletal secondary characters have not yet developed.

\section{Aims And Objectives}

This study primarily aimed to determine gender of an individual based on the Mesio-Distal (MD) dimensions of the permanent first mandibular molars on a panoramic radiograph and assess sexual dimorphism exhibited by the tooth.

\section{Materials And Method}

The present study is conducted on 100 panoramic radiographs (50 males and 50 females) of individuals between the ages of 18 and 40, obtained from archives in the department of Oral medicine \& Radiology, Yenepoya Dental College \& Hospital, Mangalore.

The inclusion criteria taken into consideration were as follows: 
- Caries free teeth.

- Presence of bilateral mandibular first molars with intact adjacent teeth.

The exclusion criteria taken into consideration were as follows:

- Missing mandibular first molars.

- Restorations and fixed orthodontic appliances.

- Supra erupted teeth .

- Overlapping of contact areas.

The panoramic radiographs used were obtained using Planmeca Promax OPG/Ceph machine that employs photostimulable phosphor plates and AGFA CR-30X scanner under standard protocols. Only those radiographs that were taken in increased interproximal function were included for this sudy. The mesiodistal width of mandibular right and left first molars were measured on panoramic radiographs using a digital scale as the maximum distance (in $\mathrm{mm}$ ) between the contact points with the mandibular second premolar and second molar teeth. (Fig 1)

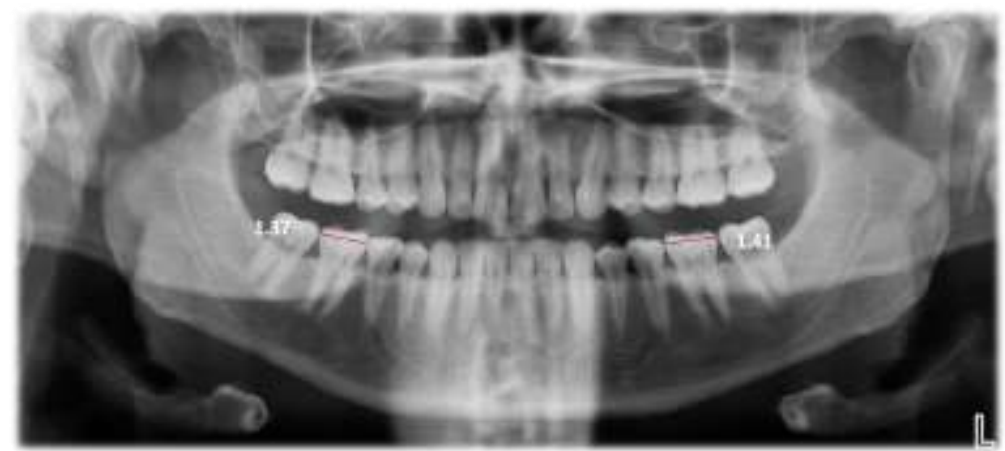

(Fig 1)

The measurements were performed by one person and all values were rounded to two decimal places. In order to assess the reliability of the measurements, intraobserver error was tested. The same measurements were obtained from 50 randomly selected radiographs from the original sample at a different time by the same observer to assess intra observer error. Another observer measured the same randomly selected radiographs in order to test the inter observer error. Their measurements were analyzed using Student's $t$-test. There was no statistically significant difference between the findings of the two observers. Statistically significant sexual dimorphisms in male and female odontometric features were tested by the unpaired $t$-test.

\section{Results And Observations :}

The mesio-distal width of right and left permanent mandibular first molars were measured by a digital scale on panoramic radiographs and were subjected to descriptive statistics [Table 1]. It was seen that the mean values of parameters did not show statistically significant differences between males and females with $P<0.05$, in the MD dimension of permanent mandibualar first molars [Table 2].

\begin{tabular}{|c|c|c|c|c|c|}
\hline \multicolumn{2}{|c|}{ Gender } & $\mathrm{N}$ & Mean & Std. Deviation & Std. Error Mean \\
\hline \multirow[t]{2}{*}{$36(\mathrm{~mm})$} & Male & 50 & 13.7080 & 1.12936 & .15972 \\
\hline & Female & 50 & 13.3540 & .91723 & .12972 \\
\hline \multirow[t]{2}{*}{$46(\mathrm{~mm})$} & Male & 50 & 14.0180 & 1.03779 & .14677 \\
\hline & Female & 50 & 13.9000 & 1.24704 & .17636 \\
\hline
\end{tabular}

Table 2: Evaluation of the relationship between mesio-distal width of 36, 46 with gender :

\begin{tabular}{|c|c|c|c|c|l|l|l|}
\hline \multicolumn{2}{|c|}{} & $\mathrm{T}$ & $\mathrm{Df}$ & Sig. & $\begin{array}{l}\text { Mean } \\
\text { Difference }\end{array}$ & $\begin{array}{l}\text { Std.Error } \\
\text { Difference }\end{array}$ & SIGNIFICANCE \\
\hline \multirow{2}{*}{$36(\mathrm{~mm})$} & & 1.720 & 98 & .088 & .35400 & .20575 & Insignificant \\
\cline { 2 - 8 } & & & & & & & \\
\hline $46(\mathrm{~mm})$ & & .514 & 98 & .608 & .11800 & .22944 & Insignificant \\
\cline { 2 - 8 } & & & & & & & \\
\hline
\end{tabular}

Since $\mathrm{p}>0.05$, there is no significant difference between males and females of MD (36 \& 46)

\section{Discussion}


Though DNA profiling gives accurate results yet measurement of linear dimensions such odontometric parameters can be used for sex assessment in a large population because they are simple, reliable, inexpensive, and easy to measure. The fact that most teeth undergo complete development before skeletal maturation makes the dentition a valuable sex indicator, particularly in young individuals. ${ }^{6}$ Hence, taking into consideration the fact that there are differences in odontometric features in particular populations, even within the similar population in the historical and evolutional context, it is necessary to determine specific population values in order to make recognition possible on the basis of dental dimensions. ${ }^{7}$

The present study tends to establish the impact of morphometry of permanent first molars in a local Mangalorean population in the assessment of gender. The comparison of mean values of parameters measured between males and females showed no statistically significant differences with $P<0.05$ in the mesio distal dimensions of the mandibular first molars.

Accordindly, the results of the present study were not in agreement with other studies done by researchers in the other parts of the world ${ }^{8,9,10,11,12}$ in which they have observed that the males had larger teeth than females in all the dimensions. In a study conducted by Zorba et al., in a Greek population, they found that males have bigger teeth than females and canine showed greatest sexual dimorphism. In their study, first molars also showed significant dimorphism which is in contrast to the present study taking into account the mandibular first molars. ${ }^{13}$

In a study conducted by Astete in Spanish and Chilean population showed that most of the teeth examined were larger in men as compared to women, with the exception of first mandibular molars in respect to MD dimension. ${ }^{14}$ The result of the present study regarding MD dimension of mandibular first molar is in agreement with the study conducted by Astete. However, they were not in agreement with the studies done by various Indian researchers who conducted similar studies in various parts of India, in which they have observed that males had larger teeth than females in all the dimensions. In a study conducted by Ramandeep Singh Narang et al in a North Indian population, it was seen that the mean values of parameters showed statistically significant differences between males and females with $P<0.05$, in the MD dimension in mandibular casts. ${ }^{2}$

In another Indian study conducted Adithi Agarwal et al on casts of 100 individuals, it was observed that the mean values of MD diameter showed statistically significant differences between males and females in mandibular first molar teeth. ${ }^{14}$

In a study conducted Sudeendra Prabhu et al on 105 Indians, it was found that the mandibular first molar was the most sexually dimorphic tooth. ${ }^{15}$

A study conducted on the odontometric gender assessment in Indians showed that the magnitude of sexual dimorphism in Indians is reduced when compared to other populations but similar to South Asian groups. ${ }^{16,17}$ The study showed that there is low univariate sexual dimorphism in Indians - only $37.5 \%$ of the entire dentition exhibited statistically larger male tooth dimensions, which is in agreement with results derived from other South Asian populations. However, the study concluded that the sex identification accuracy is relatively moderate $(75 \%)$, still relegating the dentition to being an adjunct rather than sole criteria for gender assessment.

Hence, it can be inferred that a comparison of sexual dimorphism in teeth between different populations differs among different groups. The magnitude and pattern of sexual dimorphism in size of teeth differs from one population to another and also between the generations. This variation in the magnitude of dimorphism can be a result of various factors. Some authors have explained that such variation could be due to environmental influences on tooth size. Variation in food resources exploited by different populations has been explained as one such environmental cause. Others have suggested the intrusion of cultural factors with biological forces. ${ }^{4,18}$ There can be a complex interaction between a variety of genetic and environmental factors that is responsible for the variation in the degree of dimorphism. Sex chromosomes are also known to cause different effects on tooth size. The ' $\mathrm{Y}$ ' chromosome influences the timing and rate of body development, thus producing slower male maturation, and acts additively and to a greater extent than the ' $\mathrm{X}$ ' chromosome. ${ }^{4}$ Also all the above mentioned studies were carried out either on model casts or clinically. This could have led to loss of accuracy owing to the fact that measuring the tight mesio distal contacts between teeth with a vernier calliper is quite a challenging task. In contrast to it, measurements obtained from panoramic radiographs using a digital scale, renders more accuracy. This fact has been substantiated in the literature that has proved that horizontal measurememnts done on digital OPGs are sufficiently accurate for clinical use. ${ }^{19}$ Furthermore, as a measuring tool, this software is effortless and handy to use and may be preferable for validation of radiomorphometric indices.

\section{Conclusion}

On the whole, the study concludes that sexual dimorphism of teeth is population specific and amongst the Mangaloreans , mesio distal dimensions of mandibular first molars cannot aid in gender assessment. It also 
shows that the tooth size standards based on odontometric investigations are population specific and have shown varying degree of sexual dimorphism and it is not uniform in all humans.

\section{References}

[1]. Macaluso P J Jr. Sex discrimination potential of permanent maxillary molar cusp diameters. J Forensic Odontostomatol.2010; 28(1):22 -31

[2]. Ramandeep Singh Narang, Adesh S Manchanda, Balwinder Singh. Sex assessment by molar odontometrics in North indian population. J Forensic Dent Sci. 2015 jan-apr; 7(1):54-8.

[3]. Suazo GI, Cantin LM, Lopez FB, Sandval MC.; Torres, M. S.; Gajardo R. P. \&Gajardo R. M. Sexual Dimorphism in Mesiodistal and Bucolingual Tooth Dimensions in Chilean People. Int. J. Morphol. 2008;26(3):609-614

[4]. Acharya BA, Mainali S. Univariate sex dimorphism in the Nepalese dentition and the use of discriminant functions in gender assessment. Forensic Sci Inter.2007;173:47-56.

[5]. Vahanwahal SP, Parekh DK. Study of lip prints as an aid to forensic methodology.J Indian Dent Assoc. 2000;71:269-71.

[6]. Lund H, Mornstad H. Gender determination by odontometrics in a Swedish population. J Forensic Odontostomatol 1999;17:30-4.

[7]. Iscan YM, Kedici SP. Sexual variation in bucco-lingual dimensions in Turkish dentition. Forensic Sci Int 2003;137:160-4.

[8]. Prathibha Rani R M, Mahima V G, Patil K. Bucco-lingual dimension of teeth - An aid in sex determination. J Forensic Dent Sci 2009; $1: 88-92$.

[9]. Stroud JL, Buschang PH, Goaz PW. Sexual dimorphism in mesiodistal dentin and enamel thickness. Dentomaxillofac Radiol. 1994;23:169-71.

[10]. Hattab FN, al-Khateeb S, Sultan I. Mesiodistal crown diameters of permanent teeth in Jordanians. Arch Oral Biol. 1996;41:641-5.

[11]. Rai B, Dhattarwal SK, Anand SC. Sex determination from tooth. Med Leg Update. 2008;8:3-5.

[12]. Ghodosi A, Mosharraf R, Nia FF. Sexual variation in bucco-lingual dimensions in Iranian dentition. Int J Dent Anthropol. 2008;12:1-7.

[13]. Zorba E, Moraitis K, Manolis SK. Sexual dimorphism in permanent teeth of modern Greeks. Forensic Sci Int. 2011;210:74-81.

[14]. Astete C, Valenzuela JS, Galdames IS. Sexual dimorphism in the tooth dimensions of Spanish and Chilaean peoples. Int J Odontostomatol. 2009;3:47-50.

[15]. Agrawal A, Manjunatha BS, Dholia B, Althom Y. Journal of Forensic Dental Sciences. 2015; 7(3)

[16]. Prabhu S, Acharya A B. Odontometric sex assessment in Indians.Forensic Science International Nov 2009;192:1-3

[17]. Joseph AP, Harish RK, Mohammed PK, Kumar V. How reliable is sex differentiation from teeth measurements. Oral Maxillofac Pathol J. 2013 Jan 1;4(1):289-92.

[18]. Acharya BA. Sex determination potential of buccolingual and Mesio-distal tooth dimensions. J Forensic Sci. 2008;53:790-2.

[19]. R Schulze et al..Precision and accuracy of measurements in digital radiography: J. dentomaxillofacial Radiology. 